\title{
Factores que facilitan y retrasan la invasión de coníferas exóticas en la Isla Victoria
}

\author{
María A. Relva ${ }^{\bowtie}$ \& Martín A. NuÑez \\ Laboratorio Ecotono, INIBIOMA, Conicet-Universidad Nacional del Comahue.
}

\begin{abstract}
RESUMEN. Las especies invasoras constituyen una seria amenaza a la biodiversidad y producen cambios a gran escala en las comunidades nativas y alterando los procesos de los ecosistemas. En la actualidad, los bosques Andino-Patagónicos exhiben una diversidad amplia de especies introducidas, tanto terrestres como acuáticas. Muchas de estas especies poseen un potencial invasor elevado, al tiempo que constituyen la base de diversas actividades de importancia económica para la región, como, por ejemplo, los cotos de caza de ciervos y jabalíes, la pesca deportiva de salmónidos y las forestaciones con coníferas introducidas. La situación descripta para la Patagonia brinda un escenario ideal para probar y/o generar nuevas hipótesis sobre la ecología de las invasiones. En un campo más práctico, determinar el impacto y conocer las relaciones entre las especies nativas y exóticas permitirán establecer prioridades en las acciones de manejo, así como también mitigar los efectos no deseados o inesperados como consecuencia de las tareas de erradicación o control. Presentamos aquí una síntesis de los estudios propios llevados a cabo para entender el patrón de invasión de pináceas encontrado en un bosque nativo de Nothofagus/Austrocedrus en Isla Victoria, Parque Nacional Nahuel Huapi. Entre los mecanismos estudiados encontramos que algunos de ellos la facilitan (e.g., ciervos introducidos), mientras que otros la retrasan (depredación de semillas, baja presencia de micorrizas) o no tienen un efecto sustancial en la invasión (presión de propágulos). Estos resultados y otros antecedentes disponibles a la fecha de ésta y otras regiones del mundo, permiten sugerir estrategias de manejo para impedir o retrasar una invasión masiva de pináceas, las cuales deberían posiblemente estar enfocadas a evitar nuevas introducciones, reducir las poblaciones de los herbívoros exóticos y mantener los ecosistemas nativos con bajos niveles de impacto antrópico. Al mismo tiempo, consideramos importante emprender estudios específicamente diseñados para analizar los efectos de las tareas de erradicación y/o control sugeridas.
\end{abstract}

[Palabras clave: bosque nativo, especies introducidas, facilitación, micorrizas, presión de propágulos, resistencia biótica, ungulados]

\begin{abstract}
Aвstract. Factors facilitating and hindering the invasion of exotic conifer in Isla Victoria: Invasive species constitute a serious threat to biodiversity, producing wide-scale changes in native communities and altering ecosystem processes. The Andean-Patagonian forests currently exhibit a wide diversity of terrestrial as well as aquatic introduced species. Many of these introduced species possess a high invasive potential, while at the same time have economical relevance to the region, such as sport fishing and hunting, and pine plantations. The described situation for Patagonia offers an ideal scenario to test and generate new hypotheses about the ecology of tree invasions. In a more practical domain, determining the impact and knowing the relationship between native and exotic species will permit the establishment of priorities in management actions, as well as also avoiding or mitigating the undesirable or unexpected effects that arise from eradication or control tasks. Here, we present a synthesis of our own studies carried out to understand the factors driving the pattern of pine invasion in the native Nothofagus/Austrocedrus forests on Isla Victoria, Nahuel Huapi National Park. Among studied factors, we found that some of them facilitate the invasion (e.g., introduced herbivores), while others can delay (e.g., low presence of mycorrhizae fungi, seed predation) or have neutral effects on the invasions (e.g., propagule pressure). These results and others currently available from this region and elsewhere allow us to suggest management actions to prevent or hinder a major pine invasion, which should be focused on avoiding new introductions, reducing the abundance of introduced large mammals and preserve native ecosystems with low levels of anthropic disturbances. At the same time, we consider important to start well designed studies to analyze the effects of these management actions.
\end{abstract}

[Keywords: biotic resistance, invasional meltdown, mycorrhizal, native forest, non-native, propagule pressure, ungulates]

\section{INTRODUCCIÓN}

Las especies invasoras pueden tener un enorme impacto en la biodiversidad, en el funcionamiento de los ecosistemas y en el bienestar humano (Vitousek et al. 1997; Mack et al. 2000). Los ecosistemas templados

andrearelva@gmail.com del sur de Sudamérica poseen una extensa y variada historia de introducciones de especies animales y vegetales, terrestres y acuáticas, para las cuales en su mayoría no se conoce el impacto que producen en las comunidades locales, y menos aun sobre las

Recibido: 27 de octubre de 2013; Fin de arbitraje: 16 de diciembre; Última versión: 6 de junio de 2014; Aceptado: 26 de junio. 
funciones de los ecosistemas (Jaksic 1998; Jaksic et al. 2002; Vázquez 2002; Anderson et al. 2006; Novillo \& Ojeda 2008; Merino et al. 2009). Por otro lado, algunas de estas especies introducidas y con potencial invasor poseen un alto valor económico en la región, como por ejemplo los salmónidos (Vigliano \& Alonso 2007), los ungulados introducidos como el jabalí y el ciervo colorado (Asad 2005) y las plantaciones de coníferas exóticas de crecimiento rápido (Schlichter \& Laclau 1998). Tal como ha sucedido en otras regiones del planeta, la mayoría de las especies exóticas en la Patagonia fueron introducidas de manera intencional, ya sea para fines cinegéticos (e.g., cotos de caza, pesca deportiva) o productivos (e.g., plantaciones de pináceas, cría de ganado, peletería). En consecuencia, emerge aquí un gran desafío a la hora de realizar el manejo de las especies exóticas, ya que si bien muchas de ellas son especies reconocidas como potencialmente dañinas para los ecosistemas, a su vez proveen beneficios económicos importantes para la región. Este escenario exige aun más tener un conocimiento claro de las especies introducidas, de su impacto y de las nuevas relaciones que establecen entre sí, con las especies nativas y con el ambiente receptor. En este sentido, en los últimos años se ha avanzado en el conocimiento científico acerca de los patrones de invasión y en los impactos que causan algunas de las especies introducidas e invasoras. Sin embargo, el entendimiento de los mecanismos, esto es, factores que frenan o disparan las invasiones, es a la fecha muy escaso para la mayoría de estas especies.

En la última década, el interés académico por la presencia y los efectos de las especies introducidas en la región patagónica ha aumentado de forma notable, y en los últimos tiempos ha comenzado a ser incorporado de manera más efectiva en los planes de gestión de las áreas protegidas (APN 2004, 2007). Sin embargo, la percepción que posee la población humana local sobre el impacto que las especies introducidas pueden tener sobre los ecosistemas y sobre sus actividades económicas es casi inexistente (Speziale et al. 2012) o incipiente, como pudo notarse recientemente en el caso de invasión del alga Didymosphenia geminata (moco de roca) en el lago Nahuel Huapi (Beamud et al. 2013). Sumado a esto, consideramos que aún permanecen entre los administradores de los recursos naturales y en el público en general, mitos o saberes parciales sobre las especies exóticas, sobre su estado de invasión y sobre los impactos que causan en las comunidades nativas; pensamos que estos saberes merecen ser discutidos y aclarados, a los fines de lograr un mayor entendimiento y un adecuado manejo de estas especies.

\section{Los árboles como especies invasoras}

El reconocimiento mundial de las especies leñosas (árboles y arbustos) como especies invasoras capaces de alterar los ecosistemas, es relativamente reciente (Richardson \& Rejmánek 2011). El aumento de los problemas derivados de la invasión de árboles se debe fundamentalmente al incremento rápido del transporte de especies mediado por el ser humano y a la dispersión de miles de especies, en gran medida con fines forestales y ornamentales (Simberloff et al. 2010). Entre los árboles, las especies de la familia Pinaceae son actualmente las de más amplia distribución en el mundo. Nativas casi con exclusividad del hemisferio norte, han sido introducidas y plantadas a lo largo del hemisferio sur (Australia, Sudáfrica, Nueva Zelanda y, más recientemente, Sudamérica), con fines en su mayoría comerciales, aunque también paisajísticos o para el control de la erosión del suelo. Como resultado, muchas de estas especies de pináceas, en particular pinos, han devenido en invasoras y han causado daños ecológicos y económicos graves (Richardson \& Higgins 1998). En Sudamérica el interés por estudiar las invasiones de especies de pináceas se ha incrementado en la última década (Simberloff et al. 2002, 2010; Pauchard et al. 2004, 2010; Sarasola et al. 2006; Zalba et al. 2008; Richardson et al. 2008; Orellana \& Raffaele 2010). Por otro lado, a nivel gubernamental, por ejemplo en Chile y Argentina, se fomenta la actividad al financiar el Estado gran parte de la etapa de plantación (aunque sin control posterior alguno de su dispersión en los ecosistemas adyacentes (SAGPyA 1999)). En la Patagonia Argentina, la escala de plantación en un período de tiempo corto, y con especies de pináceas reconocidas como muy invasoras en otros lugares del hemisferio sur, son factores que permiten predecir una tasa de invasión rápida en un futuro no muy lejano (Richardson et al. 2008; Simberloff et al. 2010).

Desde el inicio del estudio de la biología de las invasiones, se ha destinado un esfuerzo considerable a predecir qué hace que una especie introducida se convierta en invasora y qué características de la comunidad hacen 
que sea más o menos invadida (Elton 1958; Inderjit et al. 2005; Richardson \& Pysek 2006). Es decir, numerosas hipótesis han sido planteadas para explicar el éxito o el fracaso de las invasiones, ya sea al hacer hincapié en la especie introducida o en el sistema recipiente. En este sentido, se han alcanzado generalizaciones valiosas, aunque también abundan las excepciones. La información cualitativa de organismos no relacionados o la ausencia natural de datos en sitios donde las invasiones han fracasado, han contribuido a esta situación. Sin embargo, en algunos taxa ha habido más avances que en otros. Por ejemplo, los pinos han servido como un grupo modelo para dilucidar algunos mecanismos detrás de las invasiones de plantas (Richardson 2006). Rejmánek \& Richardson (1996) lograron separar las especies de pinos invasoras de las no invasoras, de acuerdo a tres características intrínsecas de las especies (edad reproductiva temprana, semillas livianas, gran producción de semillas a intervalos de tiempo cortos). Sin embargo, se ha señalado que este mecanismo no es suficiente para predecir las invasiones (Simberloff et al. 2002; Nuñez et al. 2011). Las hipótesis más prominentes que se han postulado para explicar el éxito variable de las invasiones de pináceas, además de las características intrínsecas de las especies, incluyen factores que operan a distintas escalas espaciales y temporales (Gundale et al. en prensa), entre ellos: la intensidad de la introducción (e.g., presión de propágulos), la interacción con factores abióticos (e.g., clima, suelo) y bióticos (e.g., herbívoros, granívoros), los disturbios (e.g., fuego, mortalidad y decaimiento por eventos climáticos) y la interacción con la comunidad biótica residente (Figura 1).

Conocer los factores y cómo operan en la invasión es relevante tanto para el avance en el conocimiento de la ecología de las invasiones como también para desarrollar programas y legislación pensados para mitigar el impacto de las invasiones. En este trabajo presentamos una síntesis de los estudios propios llevados a cabo para entender el patrón de invasión de pináceas encontrado en un bosque nativo de Nothofagus/Austrocedrus en Isla Victoria, Parque Nacional Nahuel Huapi. Los mecanismos estudiados no son los únicos que estarían contribuyendo a determinar el patrón de invasión, aunque sí, a priori, los que consideramos más probables, dada la historia natural de introducción de especies y de disturbios que posee la isla.

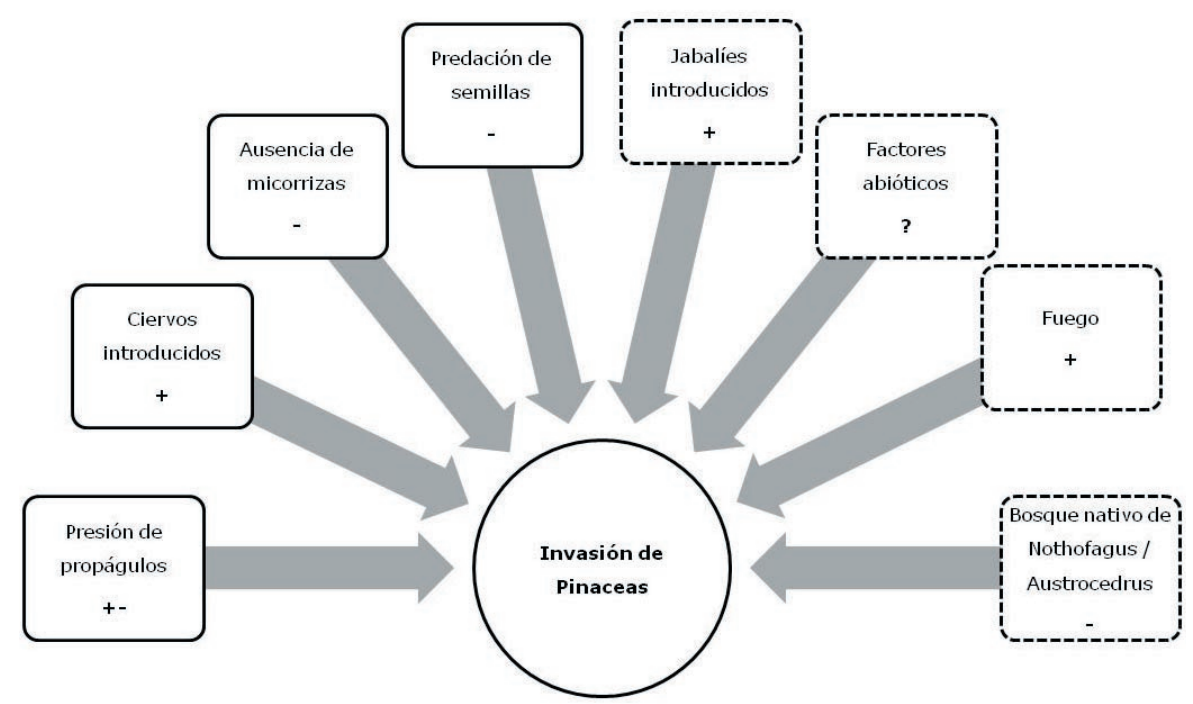

Figura 1. Modelo conceptual sobre los factores involucrados en el patrón de invasión de coníferas exóticas en el bosque nativo de Nothofagus / Austrocedrus en Isla Victoria (Parque Nacional Nahuel Huapi, Argentina). El contorno continuo de la forma indica los factores investigados en este estudio, el contorno discontinuo indica factores estudiados por otros investigadores y/o no explorados. + indica efecto de facilitación del factor sobre la invasión, - indica efecto negativo, +- indica efecto neutral, ? indica efecto desconocido.

Figure 1. Conceptual model on the factors involved in the pattern of introduced conifer invasion in the native forest of Nothofagus/Austrocedrus in Isla Victoria (Parque Nacional Nahuel Huapi, Argentina). Continuous contour in the boxes indicates the factors investigated in this study, the dashed line indicates factors studied by other researchers and / or unexplored. + Indicates facilitation effect of the factor on the invasion, - indicates negative effect, + - indicates neutral effect, ? indicates unknown effect. 
Isla Victoria: un laboratorio natural para probar hipótesis sobre invasiones biológicas

Isla Victoria es una isla de origen terciario, ubicada en el Lago Nahuel Huapi (4057' S, $71^{\circ} 33^{\prime} \mathrm{O}$ ), con una superficie de 3700 ha que se extiende de noroeste a sudeste, de $23 \mathrm{~km}$ de largo, $4 \mathrm{~km}$ en su parte más ancha y $400 \mathrm{~m}$ en su parte más angosta (área central, Puerto Anchorena) (Figura 2). A lo largo de su eje mayor, la isla está atravesada por un cordón montañoso que alcanza su altitud máxima en el Cerro Carbón (1025 m.s.n.m.), presenta valles poco profundos y varias lagunas. La precipitación media anual es 1500 mm, concentrada entre abril y septiembre, y los suelos son profundos, andisoles de textura arenosa y con buen drenaje. Isla Victoria se halla bajo la administración del Parque Nacional Nahuel Huapi (Administración de Parques Nacionales) en territorio de la provincia de Neuquén. Está cubierta por bosques dominantes puros de coihue (Nothofagus dombeyi) y ciprés de la cordillera (Austrocedrus chilensis) y bosques mixtos de ambas especies. Presenta además matorrales de radal (Lomatia hirsuta), maitén (Maytenus boaria) y ñire (Nothofagus antarctica), y pequeños bosques de arrayán (Luma apiculata) y patagua (Myrceugenia exsucca), mallines (zonas inundables durante parte del año), así como praderas abandonadas, caminos, sendas y construcciones diversas. La parte baja del bosque (sotobosque) presenta especies arbustivas como laura (Schinus patagonicus), maqui (Aristotelia chilensis), michai (Berberis heterophylla), chaura (Gaultheria sp), espino negro (Colettia hystrix), chaurilla (Maytenus chubutensis), parrilla (Ribes magallanicum); hierbas como por ejemplo amancay (Alstroemeria aurea), cacho de cabra (Osmorhiza chilensis), enredaderas (Cynamchum descolei, Mutisia decurrens y M. spinosa), helechos y gramíneas como la caña colihue (Chusquea culeou). El tipo de comunidad de plantas descriptas aquí para la isla, así como los disturbios que presentan (e.g., fuego, herbívoros introducidos, mortalidad y decaimiento por eventos climáticos) son similares a aquellas que se encuentran en la tierra firme, contigua.

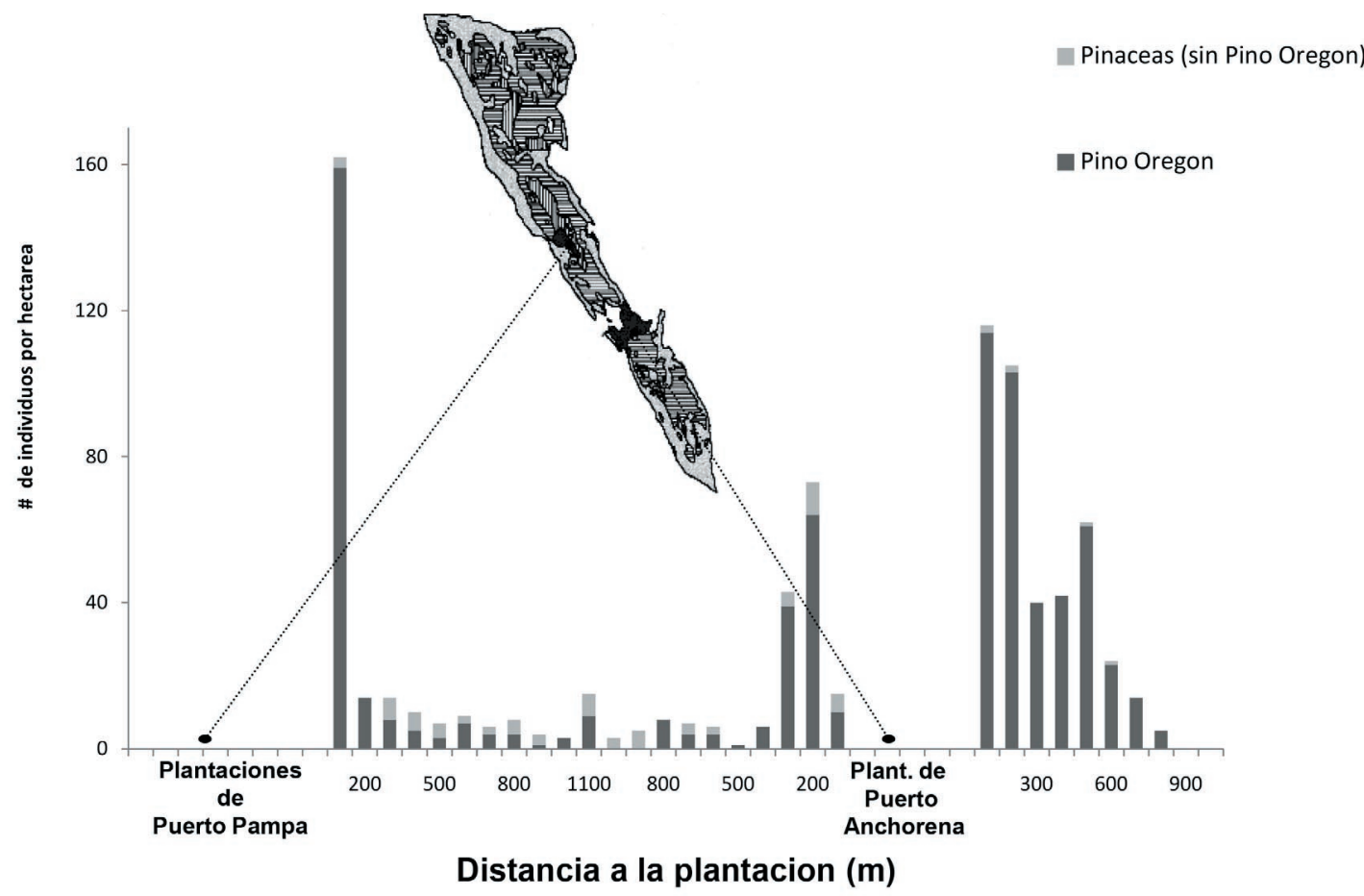

Figura 2. Densidad de plántulas, juveniles y adultos de coníferas exóticas en el bosque nativo de Nothofagus / Austrocedrus originados de dos plantaciones de aproximadamente 80 años, en el área central de la Isla Victoria (PN. Nahuel Huapi, Argentina). Pináceas (en negro) y no pináceas (en gris).

Figure 2. Density of seedlings, saplings and adults of introduced conifers in the native Nothofagus / Austrocedrus forest, originated from two 80 years old plantations in the central area of Isla Victoria (PN. Nahuel Huapi, Argentina). Pines (black) and non pines (gray). 
En el año 1925 se crea la Estación Forestal en el área central de la isla con el fin de producir especies de valor económico y estético para la región del Parque Nacional (Koutché 1942) (Figura 2). La concepción de aquella época consideraba a la flora nativa como poco diversa para los diversos usos que se requerían (especies de importancia forestal, ornamentales, frutales, cercos vivos, cortinas rompevientos) (Koutché 1942). Las especies introducidas, por el contrario, ofrecían mejores alternativas en este sentido, y se reconocía la necesidad de estudiar y experimentar con ellas; esto constituyó uno de los objetivos de la Estación Forestal. Si bien la última plantación data del año 1939-1940, funcionó como vivero hasta el año 1960. Durante ese período fueron plantadas en forma aislada y en grupos homogéneos o mixtos, al menos 71 especies de coníferas y 47 especies de latifoliadas. La superficie total forestada originalmente fue 63 ha y abarcaba tres áreas de la isla: el área central, la más extensa (Puerto AnchorenaPenínsula de Manzanito, 23 ha), y hacia el norte Puerto Pampa (26 ha) y Puerto Madera (un cuadro más pequeño, sin información disponible sobre el área implantada). Esta última plantación fue cortada totalmente en el año 1990 (A. Moretti, APN, comunicación personal). De la mayor parte de las especies cultivadas no existen registros precisos sobre el número total de individuos plantados o el área inicial ocupada por cada una de ellas (Nuñez et al. 2011). En cambio, existe información detallada sobre las tasas de crecimiento, supervivencia, producción de conos durante los primeros años, todos ellos atributos de interés forestal. Entre las especies plantadas se distinguen 21 especies del género Pinus como por ejemplo $P$. contorta, $P$. halepensis, $P$. jeffreyi, $P$. ponderosa, $P$. sylvestris (ver lista completa en Simberloff et al. (2002)).

También fueron introducidos arbustos que en la actualidad ocupan áreas extensas de la isla, como la retama (Cytisus scoparius), introducida como cortina rompe-vientos, el enebro (Juniperus communis), la rosa mosqueta (Rosa eglanteria) y la zarzamora (Rubus fructicosus), introducidas para el consumo de sus frutos. También existen poblaciones de hierbas exóticas como la dedalera (Digitalis purpurea) o la lengua de perro (Cynoglossum creticum).

Además de las especies de plantas exóticas, en el mismo período fueron introducidos en Isla Victoria ciervos de origen euro-asiático, como el colorado (Cervus elaphus) y el dama o paleto (Dama dama), los cuales utilizan (Barrios García et al. 2012) e impactan el ecosistema local con distinto grado de intensidad (Veblen et al. 1989; Relva et al. 2009, 2014). Otras especies animales introducidas en la isla y cuyo impacto aún se desconoce incluyen al visón (Mustela vison) y al faisán plateado (Lophura nycthemera). Más recientemente (ca. 1999) el jabalí europeo (Sus scrofa) arribó a la isla, aparentemente nadando (Simberloff et al. 2003). Se lo ha observado utilizando todas las comunidades de plantas y un estudio reciente revela que el disturbio en el suelo provocado por el hozado del jabalí promueve el establecimiento y biomasa de especies exóticas (Barrios Garcia \& Simberloff 2013). Por lo tanto, desde el punto de vista biológico, consideramos que la Isla Victoria emerge como un escenario o laboratorio natural ideal para poner a prueba diversas hipótesis sobre la ecología de las invasiones.

\section{Patrón de invasión de Pináceas en un bosque nativo de Nothofagus/Austrocedrus}

Observaciones previas en Isla Victoria (D. Simberloff, Universidad de Tennessee, comunicación personal) permitieron identificar a numerosas especies de coníferas exóticas allí plantadas como muy invasoras en otras partes del mundo, como Australia, Nueva Zelanda y Sudáfrica (Rejmanek \& Richardson 1996). A partir de esta observación iniciamos una serie de estudios, el primero de los cuales fue determinar si estas especies habían invadido el bosque nativo dominado por coihue (Nothofagus dombeyi) y ciprés de la cordillera (Austrocedrus chilensis). De manera contraria a lo esperado, hallamos una relativa baja invasión, excepto en ciertas áreas disturbadas o muy cercanas a la plantación (Simberloff et al. 2002, 2003) (Figura 2). En 28.73 ha de bosque nativo desde 100 metros a $1 \mathrm{~km}$ de las plantaciones, encontramos 1637 árboles introducidos, de los cuales $92.7 \%$ fueron pino oregón (Pseudotsuga menziesii) y enebro (Juniperus communis). De éstos, 90.6\% eran menores de $3 \mathrm{~m}$ de altura y su densidad disminuyó con la distancia, de manera tal que pocos individuos fueron encontrados a $0.5 \mathrm{~km}$ de la plantación y ninguno a más de $1 \mathrm{~km}$ de distancia. Si bien observaciones casuales en zonas altamente disturbadas (e.g., bordes de caminos) muestra una alta tasa de expansión de pináceas, estudios llevados a cabo en la región sobre el patrón de invasión encontraron 
un patrón similar al de nuestro estudio. Orellana \& Raffaele (2010), en plantaciones más jóvenes que las de la Isla Victoria (de hasta 35 años), encontraron altas densidades de plántulas y juveniles de pino oregón sólo en las zonas de borde $(0$ a $80 \mathrm{~m}$ de distancia de la plantación) en bosques de ciprés y matorrales del NO de la provincia de Chubut y SO de la provincia de Río Negro. Sarasola et al. (2006), también en plantaciones más jóvenes que las de la isla ( $<40$ años), relevaron numerosas plantaciones de pináceas en las provincias de Neuquén, Río Negro y Chubut, y encontraron que $P$. ponderosa no se comporta como invasora, mientras que Pseudotsuga menziesii y $P$. contorta muestran un comportamiento invasor incipiente en el bosque de ciprés y en la estepa, respectivamente. De acuerdo al concepto propuesto por Richardson et al. (2000) y aceptado por los especialistas, se considera invasión a la presencia de individuos reproductivos más allá de 100 $m$ de distancia de la plantación en menos de 50 años. Por lo tanto, de los antecedentes en tierra firme mencionados y de los propios, en Isla Victoria, no surge una evidencia clara de una invasión a gran escala de pináceas en la actualidad. Sin embargo, acorde transcurran la edad de las plantaciones y, dado el incremento en su número y el aumento en la producción de semillas, es esperable que ocurra una invasión a escala regional por especies de pináceas, en particular $P$. contorta (Simberloff et al. 2010; Pauchard et al. 2010).

Con el propósito de entender cuáles son los factores que determinan el patrón de relativa baja invasión encontrado en el bosque nativo de Nothofagus / Austrocedrus llevamos a cabo diferentes estudios en la Isla Victoria, los únicos que se realizaron en el tema hasta el momento en la isla. Abordamos aspectos relacionados a la influencia de la depredación de semillas (Nuñez et al. 2008b), a las preferencias alimenticias relativa de los ciervos exóticos por las especies arbóreas exóticas y nativas (Nuñez et al. 2008a), al rol de las micorrizas (Nuñez et al. 2009, 2013), al impacto neto de los ciervos exóticos en la abundancia y crecimiento de las especies arbóreas nativas y exóticas (Relva et al. 2010) y a la presión de propágulos (Nuñez et al. 2011). En los párrafos siguientes brindamos una síntesis de los principales resultados de los estudios y discutimos brevemente cómo estos resultados pueden ser utilizados en el manejo de estas especies.
Rol de la herbivoría en el proceso de invasión: preferencia y daño

Los mamíferos herbívoros pueden influir sobre diversos procesos ecológicos, incluyendo las invasiones biológicas (Hobbs 2001; Vavra et al. 2007). Si bien aún no se entiende con claridad los mecanismos por los cuales estos herbívoros influirían sobre la invasión de coníferas (retardo o facilitación), parecerían tener un rol relevante el tipo de comunidad invadida, la identidad de la especie de conífera y la abundancia y tipo de herbívoro (Ledgard \& Norton 2008). En el bosque, los ungulados pueden dañar los árboles por pisoteo, por consumo de brotes y ramas (ramoneo), de corteza (descortezado) y/o por el frotado de sus astas sobre los tallos, afectando su crecimiento y supervivencia (Gill 2006).

Una de las explicaciones posibles al patrón de relativa baja invasión encontrado en Isla Victoria, y basada en la hipótesis del enemigo natural (Keane \& Crawley 2002), podría ser que el daño provocado por los herbívoros (ciervos colorado y dama en este caso) limite su dispersión, en particular cuando el efecto neto negativo es mayor en las especies introducidas que en las nativas. Por el contrario, si los herbívoros dañan en mayor medida a las especies nativas, podrían beneficiar la dispersión de las introducidas por liberación de la competencia (ejemplo de la hipótesis de facilitación entre especies exóticas, "invasional meltdown", (Simberloff \& Von Holle 1999).

Para dilucidar estos interrogantes realizamos dos experimentos. En el primero de ellos efectuamos un ensayo de cafetería para evaluar preferencia, en el cual dispusimos en 38 estaciones de consumo, individuos juveniles de dos especies arbóreas nativas (coihue y ciprés) y de dos exóticas (Pinus ponderosa y Pseudotsuga menziesii), accesibles al ramoneo por ciervos (Nuñez et al. 2008a). En contra de lo esperado, al cabo de 10 semanas hallamos una preferencia clara por las especies nativas, tanto en el número de individuos consumidos $(80 \%$ en ciprés vs. $20 \%$ en pino oregón) como en la intensidad del consumo ( $25 \%$ de las ramas de coihue vs. $4 \%$ de las de pino ponderosa). En un segundo estudio evaluamos si la mayor preferencia por las especies nativas se traducía en un daño relativo mayor a las especies exóticas (Relva et al. 2010). Para ello, establecimos en un bosque de ciprés y coihue, cinco clausuras de $400 \mathrm{~m}^{2}$ (áreas donde se impidió la entrada a los ciervos) y 5 áreas adyacentes de la misma 
superficie donde los ciervos ramoneaban libremente (áreas control). Luego de 4 años de la exclusión del ciervo, las abundancias de plántulas y juveniles de la especies nativa (ciprés) y exótica (pino oregón) no difirieron entre la clausura y el control. Por otro lado, bajo ramoneo, el crecimiento relativo en altura en las especies nativas fue notablemente menor que en las exóticas. La preferencia de los ciervos exóticos por consumir las especies nativas, la rápida y positiva respuesta de la vegetación nativa y la respuesta neutral de las especies exóticas a la exclusión de ciervos exóticos sugieren que posiblemente la erradicación del ciervo en la isla ayudaría a la conservación de las comunidades nativas. Es también esperable que surjan efectos complejos de la erradicación de una especie con tantas décadas en la zona, por lo que harían falta más estudios para poder determinar los efectos directos e indirectos de la erradicación.

\section{Depredación de semillas}

La depredación de semillas puede ejercer una fuerte presión para el establecimiento de plántulas de especies invasoras y se ha sugerido como una causa importante de resistencia biótica a las invasiones. Para probar si la depredación de semillas limita el establecimiento de coníferas exóticas en esta área, se comparó la depredación de semillas en zonas cercanas a las plantaciones (colonizado por especies exóticas) y lejos de ellas (no invadidas) (Nuñez et al. 2008b). Encontramos mayor depredación de las semillas de especies exóticas por parte de especies nativas de roedores y aves, posiblemente debido a su mayor tamaño. A su vez, la depredación fue mayor en zonas alejadas de las plantaciones, lo que reduce de manera considerable las posibilidades de establecimiento de plántulas exóticas. Este estudio sugiere que los depredadores de semillas pueden ser un componente importante de la resistencia biológica a la invasión.

\section{Presión de propágulos}

Se ha sugerido recientemente que la presión de propágulos sería un factor fundamental del éxito o el fracaso de las introducciones biológicas (Lockhood et al. 2005). Pusimos a prueba esta hipótesis con los datos correspondientes a 18 especies leñosas exóticas obtenidos de los registros históricos de la isla (Nuñez et al. 2011) que se compararon con resultados de un estudio de especies y el número de individuos que actualmente invaden la isla (Simberloff et al. 2002). La presión de propágulos no predijo el actual patrón de invasión, por lo que sugerimos que otros factores tales como la resistencia biótica podrían estar operando para determinar el patrón observado de la invasión, y que la presión de propágulos puede desempeñar un papel relativamente menor en la explicación de por lo menos algunos patrones observados de éxito y fracaso de las invasiones.

\section{Rol de las micorrizas}

El rol de la biota del suelo en general ha sido propuesto para entender la dinámica de invasión (Callaway et al. 2004). Sin embargo, el rol de los mutualistas ha recibido mucha menos atención que la de los agentes patógenos. Aquí mostramos que la falta de hongos micorrícicos adecuados retrasa la invasión de pináceas exóticas al reducir tanto la probabilidad de establecimiento como de crecimiento de las especies invasoras (Nuñez et al. 2009). Con una seriede experimentos deinvernaderoy decampo encontramos niveles bajos de colonización de micorrizas y de algunas especies de hongos lejos de las plantaciones originales. Los hongos mutualistas clave se circunscriben a las áreas cercanas a las plantaciones, probablemente debido a las limitaciones para dispersarse. Los niveles bajos de inóculo lejos de las plantaciones podrían estar retrasando la invasión. Nuestros experimentos indican que las interacciones subterráneas positivas pueden desempeñar un papel clave, pero poco apreciado en la dinámica de la invasión. A su vez, más recientemente encontramos que los ciervos exóticos y los jabalíes actúan como dispersores de especies de hongos micorrízicos (en especial de hongos hipogeos del género Rhizopogon) (Nuñez et al. 2013). Esta interacción entre animales, hongos y árboles exóticos es un ejemplo claro del fenómeno llamado "invasional meltdown" (Simberloff 2006).

\section{Implicancias para el manejo}

La presencia de numerosas especies introducidas en los bosques andinopatagónicos plantea diversos desafíos para su manejo debido a que muchas de ellas tienen un alto potencial invasor combinado con un valor económico elevado. Aunque no diseñados específicamente a tal fin, algunos resultados encontrados en nuestras investigaciones podrían ser utilizados en el manejo a fin de prevenir y/o reducir el impacto de estas especies. Debido al número 
de factores que determinan la invasión de coníferas exóticas, su manejo es complejo. Aún así, consideramos que es probable que estrategias como la reducción de ungulados exóticos contribuyan a reducir la invasión. Es interesante destacar que estos resultados muestran cómo la interacción entre especies exóticas puede promover la invasión de otras especies exóticas. El efecto del jabalí ejemplifica bien este caso. Esta especie introducida hace relativamente poco tiempo (1999) parece estar promoviendo la invasión de coníferas (Barrios Garcia \& Simberloff 2013) y de hongos micorrízicos exóticos (Nuñez et al. 2013), lo cual podría haber sido minimizado si se hubiera evitado la invasión.

Otro resultado con posibles consecuencias de manejo es que los ecosistemas boscosos más prístinos ofrecerían mecanismos de freno a la invasión. Si bien este es un aspecto que merece ser estudiado más profundamente, los ecosistemas menos alterados por impacto antrópico parecen ejercer un freno biótico a la invasión de especies no-nativas (e.g., Nuñez et al. 2008b; Relva et al. 2010; Simberloff et al. $2002,2003)$. Esto sugiere que el mantenimiento de ambientes naturales con mínimos disturbios antrópicos sería una estrategia apropiada para minimizar las invasiones.

\section{SÍNTESIS}

Los estudios descriptos en este trabajo marcan con claridad la complejidad del proceso que explica el patrón de baja invasión hallada. Diferentes factores como la herbivoría por animales exóticos, la depredación de semillas, la presión de propágulos y la disponibilidad de hongos micorrízicos juegan un rol importante en explicar el patrón de relativa baja invasión e impacto de las coníferas exóticas. Además, estos factores interactúan entre sí y muchas veces producen resultados difíciles de predecir. Con la información disponible a la fecha y aun en ausencia de estudios específicos, los resultados de estos años de investigación permiten señalar la importancia de reducir la abundancia y presencia de especies exóticas, y de mantener los ambientes naturales con mínimo disturbio antrópico como estrategias preventivas para limitar las invasiones de especies exóticas.

Agradecimientos: Agradecemos especialmente a A. Valenzuela y a los revisores del manuscrito por sus aportes en la primera versión del manuscrito que ayudaron a mejorarlo. Por la misma razón agradecemos a la editora de Ecología Austral, MSemmartin. Parte de estos estudios fueron financiados por la Universidad Nacional del Comahue y el Consejo Nacional de Investigaciones Científicas y Técnicas de la Argentina. Agradecemos a la Intendencia del Parque Nacional Nahuel Huapi y al cuerpo de Guardaparques de Isla Victoria por el apoyo brindado en la logística de los estudios, a la empresa Espacio SRL por facilitar el traslado de los investigadores a Isla Victoria y a la Hostería Nacional Isla Victoria por el financiamiento parcial de los experimentos.

\section{BIBLIOGRAFÍA}

ANDERSON, CB; R RozZi; JC TORRES-Mura; SM McGeHEe; MF SHERRIFFs; ET AL. 2006. Exotic vertebrate fauna in the remote and pristine sub-Antarctic Cape Horn Archipelago, Chile. Biodivers. Conserv., 15:3295-3313.

Administración de Parques Nacionales (APN). 2004. Prioridades para la investigación científica y el desarrollo de información en la Administración de Parques Nacionales. Informe Técnico APN. Buenos Aires. Pp. 20.

Administración de Parques Nacionales (APN). 2007. Lineamientos Estratégicos para el Manejo de Especies Exóticas en la APN. Informe Técnico APN. Buenos Aires. Pp. 45.

AsAD, A. 2005. Carne de ciervo, un negocio refinado. Informe Técnico, Dirección de Industria Alimentaria, SAGPyA. Buenos Aires. Pp. 3.

Barrios-García, M; MA Relva \& T Kitzberger. 2012. Patterns of use and damage by exotic deer on native plant communities in northwestern Patagonia. Eur. J. Wildl. Res., 58:137-146.

Barrios García, M \& D Simberloff. 2013. Linking the pattern to the mechanism: How an introduced mammal facilitates plant invasions. Austral Ecol., 38:884-890.

Beamud, G; G Baffico; F Pedrozo \& M Díaz. 2013. First record of the invasive algae Didymosphenia geminata in the lake Nahuel Huapi: Argentina, Patagonia. Revista Chilena de Historia Natural, 86:493-496.

Callaway, RM; GC Thelen; A Rodríguez \& WE Holben. 2004. Soil biota and exotic plant invasion. Nature, 427:731-733.

ElTON, CS. 1958.The Ecology of invasions by animals and plants. The University of Chicago Press, EEUU. Pp. 179.

GiLL, R. 2006. The influence of large herbivores on tree recruitment and forest dynamics. Pp. 170-202 en: Danell, K; R Bergstrom, P Duncan P\& J Pastor (eds.). Large Herbivore Ecology, Ecosystem Dynamics and Conservation. Vol. Conservation Biology, 11. Cambridge University Press, Cambridge.

Gundale, MJ; A Pauchard; B Langdon; DA Peltzer; BD MAXWELL; ET AL. En prensa. Can model species be used to advance the field of invasion ecology? Biol Invasions. doi 10.1007/s10530-013-0610-0.

Hоввs, R. 2001. Synergisms among Habitat Fragmentation, Livestock Grazing, and Biotic Invasions in Southwestern Australia. Conserv. Biol., 15:1522-1528.

INDERJIT, M CADOTTE \& R COLAUTtI. 2005. The Ecology of biological invasions: past, present and future. Pp. 1943 en: Inderjit, $\mathrm{M}$ (ed.). Invasive Plants: Ecological and Agricultural Aspects. Birkhauser-Verlag AG, Basel.

JAKSIC, FM. 1998. Vertebrate invaders and their ecological impacts in Chile. Biodivers.Conserv., 7:1427-1445.

JAKSIC, FM; J Agust; JE JiM \& DR MART. 2002. Invaders without frontiers: cross-border invasions of exotic mammals. Biol. Invasions, 4:157-173.

KeANe,RM\&MJ Crawley. 2002. Exotic plant invasions and the 
enemy release hypothesis. Trends Ecol. Evol., 17:164-170.

Koutché, V. 1942. Estación Forestal de Puerto Anchorena, Isla Victoria; su organización y trabajos. Informe Técnico, Ministerio de Agricultura, Dirección de Parques Nacionales, Buenos Aires, Argentina.

LEDGARD, N \& D NORTON. 2008. The impact of browsing on wilding conifers in the south island high country. New Zeal. J. For., 52:29-34.

LockHood, JL; P CAssey \& TM Blackburn. 2005. The role of propagule pressure in explaining species invasions. Trends Ecol. Evol., 20:223-228.

Mack, R; D Simberloff; W Lonsdale; H Evans; M Clout; ET AL. 2000. Biotic Invasions: Causes, Epidemiology, Global Consequences, and Control. Ecol. App., 10:689-710.

Merino, ML; BN Carpinetti \& AM AbBa. 2009. Invasive mammals in the National Park System of Argentina. Nat. Area J., 29:42-49.

Novillo, A \& R OJEDA. 2008. The exotic mammals of Argentina. Biol. Invasions, 10(8):1333-1344.

NuÑEZ MA; J HAYWARD; TR HoRTon; GC AMICO; RD Dimarco: ET AL. 2013. Exotic mammals disperse exotic fungi that promote invasion by exotic trees. Plos One, 8: e66832.

NuÑEZ, MA; TR Horton \& D SimberLoff. 2009. Lack of belowground mutualisms hinders Pinaceae Invasions. Ecology, 90:2352-2359.

Nuñez, MA; A Moretti \& D Simberloff. 2011. Propagule pressure hypothesis not supported by an 80-year experiment on woody species invasion. Oikos, 120:1311-1316.

NuñeZ, MA; MA Relva \& D Simberloff. 2008a. Enemy release or invasional meltdown? Deer preference for exotic and native trees on Isla Victoria, Argentina. Austral Ecol., 33:317-323.

Nuñez, MA; D Simberloff \& MA Relva. 2008b. Seed predation as a barrier to alien conifer invasions. Biol. Invasions, 10:1389-1398.

OreLlana, IA \& E RAFFaeLE. 2010. The spread of the exotic conifer Pseudotsugamenziesii in Austrocedrus chilensis forests and shrublands in northwestern Patagonia, Argentina. New Zeal. J For. Sci., 40:199-209.

Pauchard, A; MA Nuñez; E Raffaele; RO Bustamante; N LEDGARD; ET AL. 2010. Introduced conifer invasions in South America: an update. Front. Biogeography, 22:34-36.

Pauchard, A; LA Cavieres; RO Bustamante; PJ Becerra \& EH RAPOPORT. 2004. Increasing the understanding of plant invasions in southern South America: first symposium on Alien Plant Invasions in Chile. Biol. Invasions, 6:255-257.

REJMÁNEK, M \& DM RICHARDSON. 1996. What attributes make some plant species more invasive? Ecology, 77:1655-1661

RelVA, MA; C López Westerholm \& T KitZBERGer. 2009. Effects of introduced ungulates on forest understory communities in northern Patagonia are modified by timing and severity of stand mortality. Plant Ecol., 201:11-22.

Relva, MA; MA NuÑEZ \& D SimberLofF. 2010. Introduced deer reduce native plant cover and facilitate invasion of non-native tree species: evidence for invasional meltdown. Biol. Invasions, 12:303-311.

ReLVA, MA; E CASTÁN \& MJ MAZZARINO. 2014. Litter and soil properties are not altered by invasive deer browsing in forests of NW Patagonia. Acta Oecologica, 54:45-50

RichaRdson, D \& R Higgins.1998. Pines as invaders in the southern hemisphere. Pp. 450-473 en: Richardson, DM (ed.). Ecology and biogeography of Pinus.Cambridge University Press, Cambridge.
Richardson, DM; P PYSEK; M RejMÁNeK; MG BARBour; FD PANETTA; ET AL. 2000. Naturalization and invasion of alien plants: concepts and definitions. Divers. Distrib., 6:93-107.

RICHARDSON, DM. 2006. Pinus: a model group for unlocking the secrets of alien plant invasions? Preslia, 78:375-388

RichARDSON, DM \& P PYSEK. 2006. Plant invasions: merging the concepts of species invasiveness and community invasibility. Progress in Physical Geography, 30:409-431.

Richardson, DM; B van Wilgen \& M Nuñez. 2008. Alien conifer invasions in South America: short fuse burning? Biol. Invasions, 10:573-577.

RICHARDSON, DM \& M REJMÁNEK. 2011. Trees and shrubs as invasive alien species - a global review. Divers.Distrib., 17:788-809.

SARASOLA, MM; VE Rusch; TM SChlichter \& CM GHERSA. 2006. Invasión de coníferas forestales en áreas de estepa y bosques de ciprés de la cordillera en la región AndinoPatagónica. Ecol. Austral, 16:143-156.

Schlichter, T \& P Laclau.1998.Ecotono estepa-bosque y plantaciones forestales en Patagonia norte. Ecol. Austral, 8:285-296.

Secretaría de Agricultura, Ganadería, Pesca y Alimentación (SAGPYA). 1999. Argentina, oportunidades de inversión en bosques cultivados. Informe Técnico. Pp. 208.

SimberLofF, D. 2006.Invasional meltdown 6 years later: important phenomenon, unfortunate metaphor, or both? Ecol. Lett., 9:912-919.

Simberloff, D; MA Nuñez; NJ Ledgard; A Pauchard; DM RICHARDSON; ET AL. 2010. Spread and impact of introduced conifers in South America: Lessons from other southern hemisphere regions. Austral Ecol., 35:489-504.

Simberloff, D; MA Relva \& MA NuÑEZ. 2002. Gringos en el bosque: Introduced tree invasion in a native Nothofagus / Austrocedrus forest. Biol. Invasions, 4:35-53.

SimberlofF, D; MA Relva \& MA Nuñez. 2003. Introduced species and Management of a Nothofagus/Austrocedrus forest. Environ. Manage., 31:263-275.

SimberlofF, D \& B Von Holle. 1999. Positive interactions of non indigenous species: invasional meltdown? Biol. Invasions, 1:21-32.

Speziale, K; S Lambertucc;; MCarrete \& J Tella. 2012. Dealing with non-native species: what makes the difference in South America? Biol. Invasions, 14:1609-1621.

VÁzQuEZ, D. 2002. Multiple effects of introduced mammalian herbivores in a temperate forest. Biol. Invasions, 4:175-191.

Veblen TT; M Mermoz; C Martin \& E Ramilo. 1989. Effects of exotic deer on forest regeneration and composition in northern Patagonia. J. App. Ecol., 26:711-724.

VigLiAno, PH \& MF AlONSO. 2007. Salmonid introductions in Patagonia: a mixed blessing. Pp. 315-331 en: Bert, TM (ed.). Ecological and Genetic Implications of Aquaculture Activities. Vol 6. Reviews: Methods and Technologies in Fish Biology and Fisheries. Springer Press.

VAVRA, M; C PARKS \& M WISDOM. 2007. Biodiversity, exotic plant species, and herbivory: The good, the bad, and the ungulate. For. Ecol. Manag., 246:66-72.

Vitousek PM; CM Dantonio; LL Loope; M RejManeK \& R WestBROOKS. 1997. Introduced species: A significant component of human-caused global change. New Zeal. J. Ecol., 21:1-16.

ZALBA, SM; YA Cuevas \& RM Boó. 2008. Invasion of Pinus halepensis Mill. following a wildfire in an Argentine grassland nature reserve. J. Env. Manag., 88:539-546. 